\title{
Bilateral optic neuropathy as a complication of allogeneic hematopoietic stem cell transplantation
}

\author{
Jan Lestak ${ }^{\mathrm{a}}$, Petr Mlcak ${ }^{\mathrm{b}, \mathrm{c}}$, Adam Kuba ${ }^{\mathrm{d}}$, Klara Maresovab ${ }^{\mathrm{b}}$, Martin Kyncla, Jaroslav Tintera ${ }^{\mathrm{a}}$, Martin Fus ${ }^{\mathrm{a}}$
}

Background. The authors present a case study which describes the development of bilateral optic neuropathy as a complication of allogeneic hematopoietic stem cell transplantation (HSCT) in a patient who underwent a transplant for B-cell acute lymphoblastic leukemia (B-ALL). The patient, who was in remission with regard to the underlying hematological disease, developed edema of both optic discs and maculas three months after transplantation. The morphological finding regressed after treatment with corticoids and comprehensive systemic anti-infective therapy. However, the loss of function was not entirely restored.

Case Report. One year after the healing, the atrophy of the optic discs persisted, with corresponding findings in vessel density (VD), retinal nerve fibre layer (RNFL) and visual field changes. Electrophysiological examination by pattern electroretinogram (PERG) showed an alteration in retinal ganglion cells in the left eye, but with significant damage to nerve fibres on both sides. Visual evoked potential (VEP) verified bilateral non-inflammatory neurogenic lesions. This finding was also confirmed by functional magnetic resonance imaging (fMRI). Examination by structural magnetic resonance imaging (MRI) showed inflammatory changes in the optic nerve sheaths over time and a consequent marked narrowing of them.

Conclusion. The authors believe that edema of the optic discs and maculas was caused by a combination of several factors. Firstly, MRI showed inflammatory changes in the optic nerve sheaths, which led to a blockade of axoplasmic transport. Another factor that may have played a part in the outcome was endothelial damage to blood vessels with impaired microcirculation supplying the optic nerve fibres, which contributed to the occurrence of macular edema.

Key words: optic disc, macula, edema, lymphoblastic leukemia, hematopoietic stem cell, allogeneic transplantation, MRI

Received: September 9, 2021; Revised: September 17, 2021; Accepted: September 17, 2021; Available online: September 30, 2021 https://doi.org/10.5507/bp.2021.056

(c) 2021 The Authors; https://creativecommons.org/licenses/by/4.0/

aJL Opthalmology Clinic, Faculty of Biomedical Engineering, Czech Technical University in Prague, Czech Republic

${ }^{b}$ Department of Ophthalmology, Faculty of Medicine and Dentistry, Palacky University and University Hospital Olomouc, Czech Republic 'Department of Physiology, Faculty of Medicine and Dentistry, Palacky University Olomouc, Czech Republic

${ }^{d}$ Department of Hemato-Oncology, Faculty of Medicine and Dentistry, Palacky University and University Hospital Olomouc, Czech Republic Corresponding author: Jan Lestak, e-mail: lestak@seznam.cz

\section{INTRODUCTION}

Allogeneic hematopoietic stem cell transplantation (HSCT) is often the only treatment modality for a range of malignant and non-malignant hematopoietic disorders. Despite considerable advances in transplantation approaches, HSCT is encumbered with transplant morbidity and mortality.

Vascular endothelial dysfunction is the pathophysiological basis for a number of transplant complications such as graft-versus-host disease (GVHD), sepsis, sinusoidal obstruction syndrome and transplant-associated thrombotic microangiopathy (TA-TMA) (ref. ${ }^{1}$ ).

A number of factors play a part in endothelial damage, from anti-tumor and immunosuppressive therapy, through infectious complications, to immunopathological processes associated with GVHD (ref..$\left.^{2-4}\right)$.

Neurological complications present a heterogeneous range of transplant morbidities in the early and later periods after HSCT. The most common causes of this variety of complications include neuroinfections, a recurrence of the underlying disease, drug toxicity, cerebrovascular events, metabolic disorders and immunopathological processes. The frequency of neurological complications of non-infectious etiology ranges from $7.5 \%$ at 3 months after transplantation to $13 \%$ at 5 years after transplantation ${ }^{5}$.

In the first days and weeks after an allogeneic transplant, the most common causes of neurological complications are drug toxicity (chemotherapy, immunosuppressive therapy), metabolic reasons, and bacterial, fungal and viral infections. In the period commencing three months after transplantation, the characteristic pathogenetic processes are neurological complications of TA-TMA, the reactivation of opportunistic viruses, chronic GVHD and the recurrence of the underlying disease ${ }^{6}$.

Inflammatory impairments of the optic nerve are a rare neurological complication of allogeneic transplantation. There have been case studies that describe associations with reactivations of certain opportunistic viruses, GVHD, or immune-mediated demyelinating diseases ${ }^{7-11}$.

In the following case study, we present a form of optic neuropathy of combined etiology as a possible con- 
sequence of endothelial dysfunction and inflammatory neuropathy in the field of deregulated immune system function after allogeneic transplantation.

\section{CASE REPORT}

The patient (born 1984) did not have eye problems in childhood. In September 2018, he was diagnosed with Ph-negative B-cell acute lymphoblastic leukemia (B-ALL) without central nervous system (CNS) involvement. The patient underwent treatment according to GMALL junior protocol, including repeated prophylactic intrathecal applications of cytostatics, without serious complications. He underwent regular ophthalmological examinations before lumbar punctures and the ophthalmological findings were always normal.

The patient achieved hematological remission, but, due to the persistence of minimal residual disease in the bone marrow, he was indicated for allogeneic HSCT. In March 2019, the patient underwent allogeneic HSCT from an HLA-matched unrelated donor after a reduced intensity conditioning (fludarabine $150 \mathrm{mg} / \mathrm{m} 2$, melphalan $140 \mathrm{mg} / \mathrm{m} 2$, anti-thymocyte globulin $4 \mathrm{mg} / \mathrm{kg}$ ). Prior to and during the preparation regimen, the patient was prophylactically treated with valganciclovir for previous mild symptoms of sinusitis along with the detection of human herpes virus-6 (HHV-6) from a nasopharyngeal swab. For GVHD prophylaxis, cyclosporin A was used at a dose of $3 \mathrm{mg} / \mathrm{kg}$ every $12 \mathrm{~h}$ with an initial target serum level of $250-300 \mu \mathrm{g} / \mathrm{L}$ and a planned dose reduction from day +30 until complete withdrawal around day +100 to +120 after transplantation. The early post-transplant course was complicated by the development of febrile neutropenia. Despite the normalization of the inflammatory laboratory parameters after three weeks of antibiotic and antifungal therapy, subfebrile symptoms persisted in the clinical picture and a new discrete skin rash developed. Examination of the bone marrow on day +21 revealed a significant viral load of HHV-6, the replication of which was also detected slightly in the peripheral blood. Anti-infective therapy was enforced by the virostatic treatment with foscarnet, which led to a gradual reduction in clinical symptoms. After transplantation, the patient achieved remission of B-ALL, including complete minimal residual disease negativity in the bone marrow at $100 \%$ of the donor's chimerism. In the following course, the patient was monitored on an outpatient basis without complications and without any manifestations of GVHD. Systemic immunosuppression was tapered according to protocol.

Around day +70 , the patient developed a respiratory infection of a sino-bronchitis-like character with febrile periods, which was managed on an outpatient basis using macrolide antibiotics (bacterial, mycological and virological examinations negative, high-resolution computed tomography of the lung negative). On day +91 , the patient was hospitalised for non-specific pressure behind the eyes and blurred vision, which he had experienced for around a week. The initial magnetic resonance imaging (MRI) of the brain found a marginally increased saturation of both optic nerves and the intracranial finding was without pathology.

Immunophenotyping of cerebrospinal fluid ruled out CNS infiltration. Reactive CD8+ T lymphocytes were detected in the cerebrospinal fluid. The biochemical analysis of the cerebrospinal fluid was dominated by increased intrathecal synthesis of $\operatorname{IgG}$ and IgM of a polyclonal character, as well as increased amounts of albumin and glucose. The immunocompromised patient was put on an empirically initiated complex therapy against opportunistic neuroinfections, which included antiviral treatment with foscarnet and anti-toxoplasma treatment. The microbiological examinations of the cerebrospinal fluid were all negative, including PCR (CMV, EBV, HHV-6, PB19, ADV, BK virus, enteroviruses, JC virus, HSV-1, HSV-2, VZV, Toxoplasma, Borrelia, Aspergillus, Candida, Cryptococcus).

On 21 June 2019, the central visual acuity (CVA) of the right eye was 0.25 with a correction of $+2.0 \mathrm{D}$. The CVA of the left eye of 0.67 correction was unaffected. The slit lamp biomicroscopy finding was normal in the anterior segment.

At the posterior pole of the right eye, the optic disc had a massive pale edema, covered with numerous soft exudates and innumerable hemorrhages. All the vessels were immersed, the edema flowed from the optic disc to the macula, and the retina was attached, without focal changes. There was massive overpressure in the veinous system and the arteries were narrowed.

The finding at the posterior pole of the left eye was similar to that of the right. The optic disc had a massive pale edema, slightly smaller than that of the right eye, which was covered with numerous soft exudates and hemorrhages, and all the vessels were immersed. The macula was without obvious edema and the retina was attached, without focal changes. There was massive dilatation of the veins and the arteries were narrowed.

The optical coherence tomography (OCT) was conducted by Spectralis ${ }^{\circledR}$ OCT (Heidelberg Engineering $\mathrm{GmbH}$, Heidelberg, Germany).

In the right eye, OCT demonstated massive optic disc edema and diffuse macular edema especially nasally with reduction of foveal depression and subfoveal serous neuroepithelium detachment (Fig. 1). In the left eye, there were diffuse optic disc edema and macular edema nasally significant but less visible than on the right, a foveal depression only slightly shallowed and a small parafoveal nasal serous neuroepithelium detachment (Fig. 2).

The finding was concluded to be bilateral intraocular and retrobulbar optic neuritis and macular edema in both eyes.

Despite the complex anti-infective therapy that was administered, the optic disc edema progressed in the course of a few days, resulting in bilateral amaurosis and limited left eye abduction on 26 June 2019. The pupils were in moderate mydriasis, without photoreaction. With the agreement of the hemato-oncologists, intravenous corticoid therapy (initially dexamethasone $16 \mathrm{mg} /$ day) was administered with a concomitant withdrawal of systemic GVHD prophylaxis (day +97$)$. The effect of the treatment 


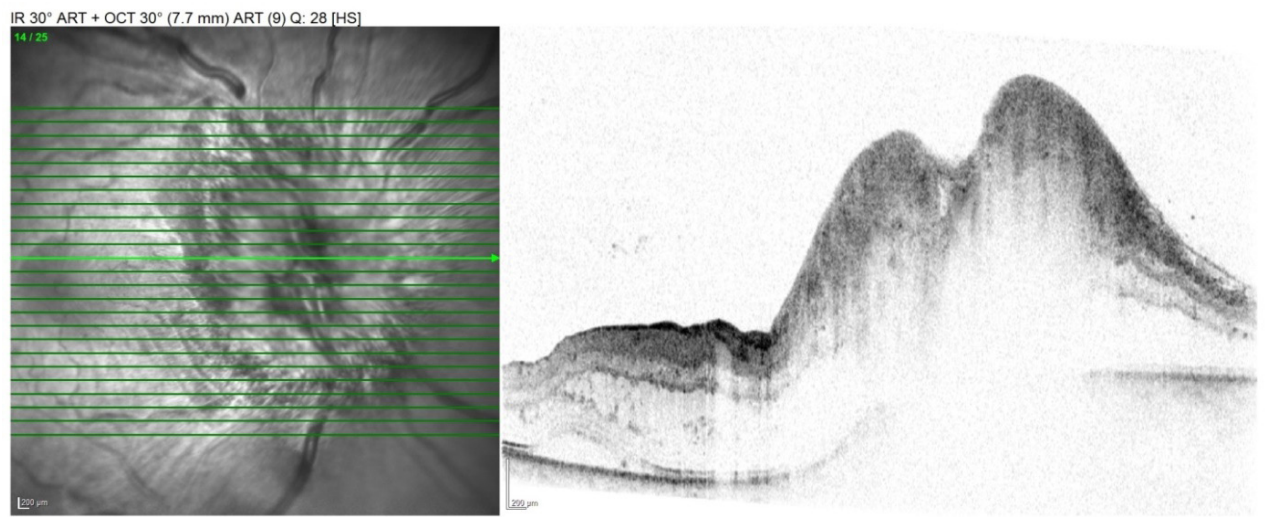

Fig. 1. OCT of the right eye conducted on 21 June 2019. Preview image (left) and linear horizontal scan (right) showing massive optic disc edema and diffuse macular edema nasally with reduction of foveal depression and subfoveal serous neuroepithelium detachment (blue arrow).

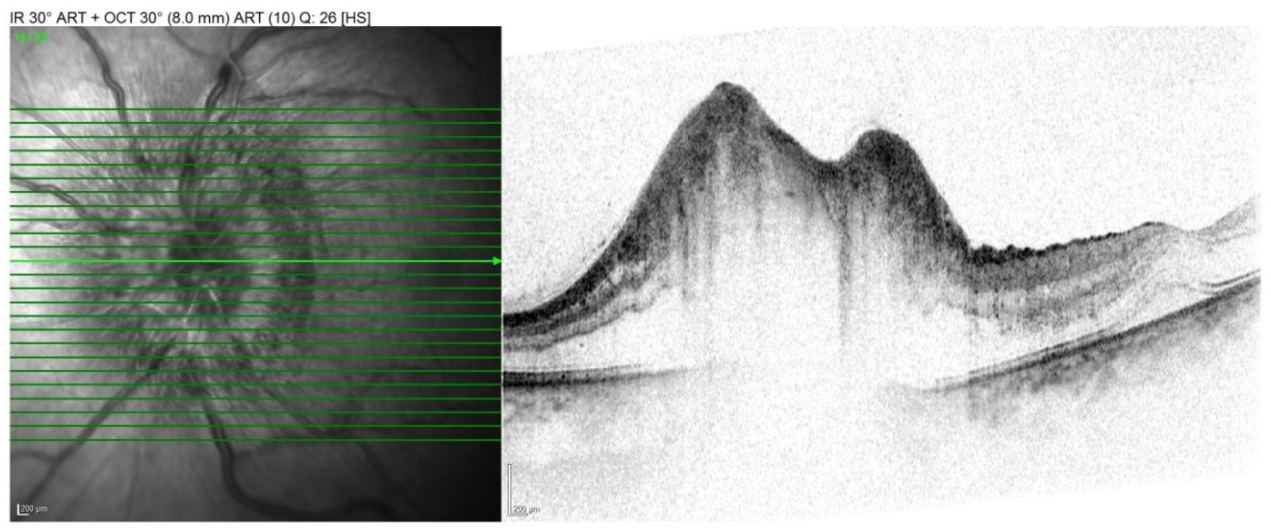

Fig. 2. OCT of the left eye conducted on 21 June 2019. Preview image (left) and linear horizontal scan (right) showing diffuse optic disc edema, macular edema nasally, a foveal depression only slightly shallowed and a small parafoveal nasal serous neuroepithelium detachment (blue arrow).

was apparent within two days. On 28 June 2019, eyesight in the right eye was partially restored (CVA 0.025), while partial restoration of eyesight in the left eye occurred on 1 July 2019 (CVA 0.025). At the same time, there was a significant reduction of optic disc edema and hemorrhages in the both eyes.

In the following period, the patient was monitored on an outpatient basis. In the search for possible causes of the condition, the patient tested positive for ANA and ENA autoantibodies. Mild fibrinolysis with no requirement for supplementation was observed in the coagulation parameters. On day +126 , the patient developed deep vein thrombosis of the right lower limb. At the same time, lupus anticoagulant positivity was repeatedly detected. Nevertheless, a possible antiphospholipid syndrome was ruled out. The patient did not develop any signs of GVHD activity.

During an examination on 25 July 2019, the CVA was 0.4 for the right eye and 0.32 for the left. The following slit lamp biomicroscopy findings were in both eyes: unstable tear film (break-up time (BUT) 1 second), marked reduction of tear meniscus, smooth cornea, clear anterior chamber, round responsive pupil, but left photoreaction was still limited, and clear lens.
At the posterior pole of the right eye, the optic disc was almost completely visible with normal colour inferotemporally, otherwise paler, around the residual exudates, a small splinter-like preretinal hemorrhage, the encapsulation of some papillary vessels, the fovea of a coarse structure, small hard regressing drusen in the fovea and around it. The retina was free of hemorrhages and attached, a slight dilatation of the veins was still evident, and the arteries were narrowed.

On the left eye, the optic disc was small, markedly pale, around it there were residual exudates and small splinter-like preretinal hemorrhages, and also clusters of peripapillary blot subretinal hemorrhages. The encapsulation of some vessels on the optic disc was also evident. The fovea was coarse in structure with small hard regressing drusen in and around it. Otherwise, the retina was without hemorrhages and attached, dilatation of the veins was still evident, more than on the right eye, and the arteries were narrowed. OCT showed further regression of the optic disc edema on the both sides, a reduction in diffuse retinal leakage and exudations being absorbed in the fovea.

On 26 August 2019, there had been a further reduction in edema and there were already signs of incipient atrophy 


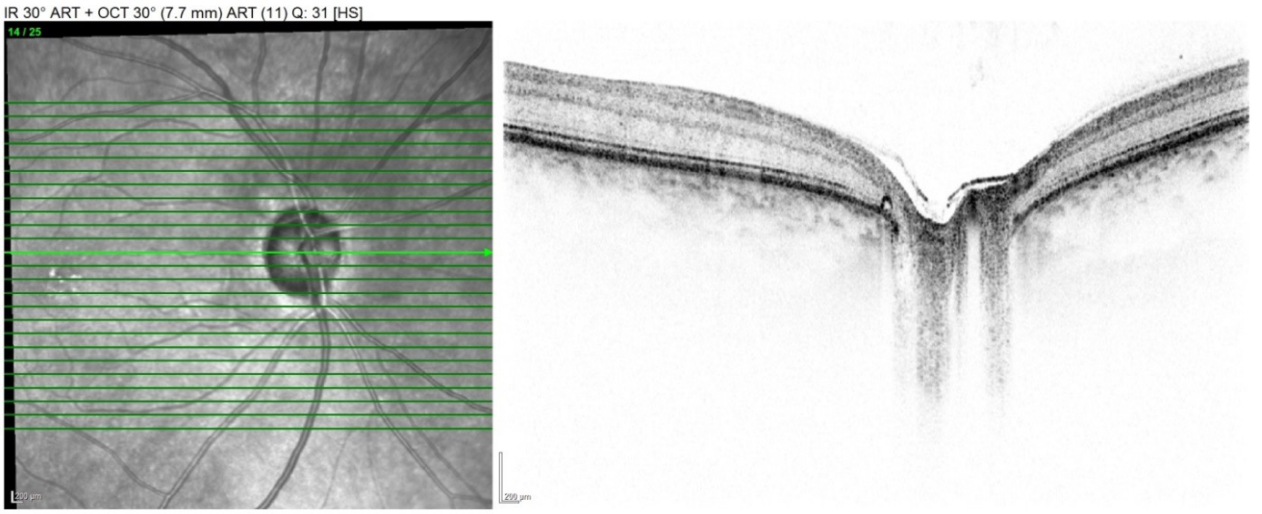

Fig. 3. OCT of the right eye from 22 September 2020. Preview image (left) and linear horizontal scan (right) showing relatively marked optic disc atrophy.

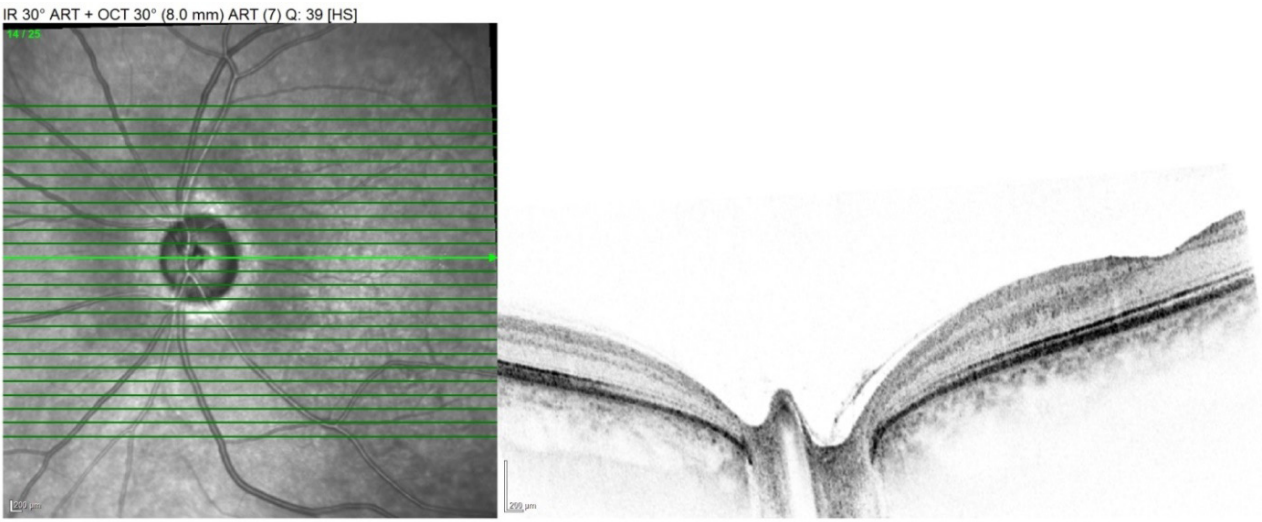

Fig. 4. OCT of the left eye from 22 September 2020. Preview image (left) and linear horizontal scan (right) showing marked optic disc atrophy and mild macular atrophy.

of the retinal nerve fibre layer. The CVA of the right and left eye was 0.5 and 0.32 respectively.

Corticoids were gradually tapered in an attempt to complete withdrawal. However, around day +160 , while the patient was already on residual and intermittent doses of corticoids, recurrence of febrile periods developed and subjective deterioration of CVA occurred (despite an unchanged ophthalmological finding). Doses of corticoids were therefore increased (prednisone $1 \mathrm{mg} / \mathrm{kg}$ ), leading to swift subjective improvement. In the following period, full anticoagulation was continued ( 9 months in total) and corticoids were gradually tapered with complete cessation on day +384 .

On 16 September 2019, with the progression of atrophy and at the request of the patient, supportive therapy was indicated for both eyes, i.e., betaxolol twice per day and supplements perorally (a total daily dose of ginkgo biloba extract of $120 \mathrm{mg}$ ). During this treatment, the narrowed retinal vessels gradually widened at the optic disc and the area around it.

During the final examination on 22 September 2020, the CVA was 0.63 for both left and right eyes. Atrophy of the optic disc was without progression and persisted positive findings on the retinal vessels (Fig. 3 and 4). A visual field examination (Octopus ${ }^{\circledR} 900$ perimeter, Haag-Streit AG, Köniz, Switzerland) had repeatedly low validity. An irregular reduction in sensitivity remained for the right eye. In the left eye, there were only small central and paracentral remnants of the visual field at the top (Fig. 5 and 6$)$.

After this, the patient was symptom free, but 18 months after the transplant, there was a second occurrence of deep veinous thrombosis in the lower part of the left limb. At the same time, a congenital prothrombotic state of APC resistance was detected. Consequently, continuous anticoagulant therapy with rivaroxaban was instituted by the patient's general practitioner.

Since the damage to both optic nerves, the patient has been in remission for B-ALL almost 2 years after transplantation. He is regularly monitored by a hematologists and an ophthalmologists.

Ocular findings from September 2020: CVA right eye 0.6 , left eye 0.6 partly; right / left eye intraocular pressure $10 / 13 \mathrm{mmHg}$; colour vision was good in the right eye, achromatopsia in the left; the anterior segment of both eyes was normal, only a relative afferent pupillary defect in the left eye persisted; on the fundus of the right eye there was an atrophic optic disc, the vascular encapsulation was only residual, the macula was without edema with retinal pigment epithelium changes, a slight dilation of the inferior temporal vein; on the left eye, the atrophy of the optic disc was more pronounced, the macula 


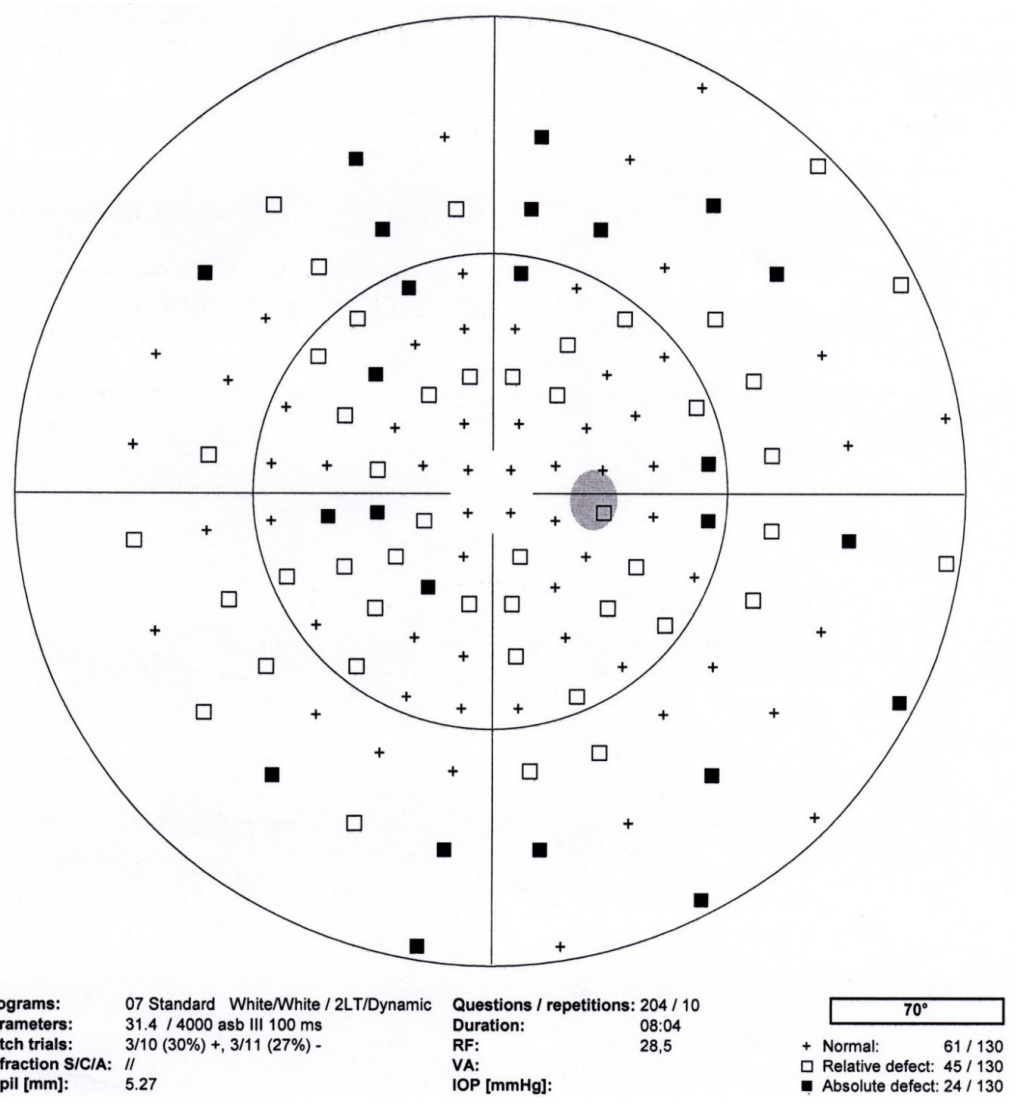

Fig. 5. Visual field examination of the right eye from 22 September 2020. In addition to the dominant points with preserved sensitivity (especially in the centre and paracentral, denoted by the symbol +), diffused more prevalent relative scotomas (especially in the paracentral, symbol $\square$ ) are visible, as are the least frequent absolute scotomas (especially in the periphery, symbol $\mathbf{\square}$ ).

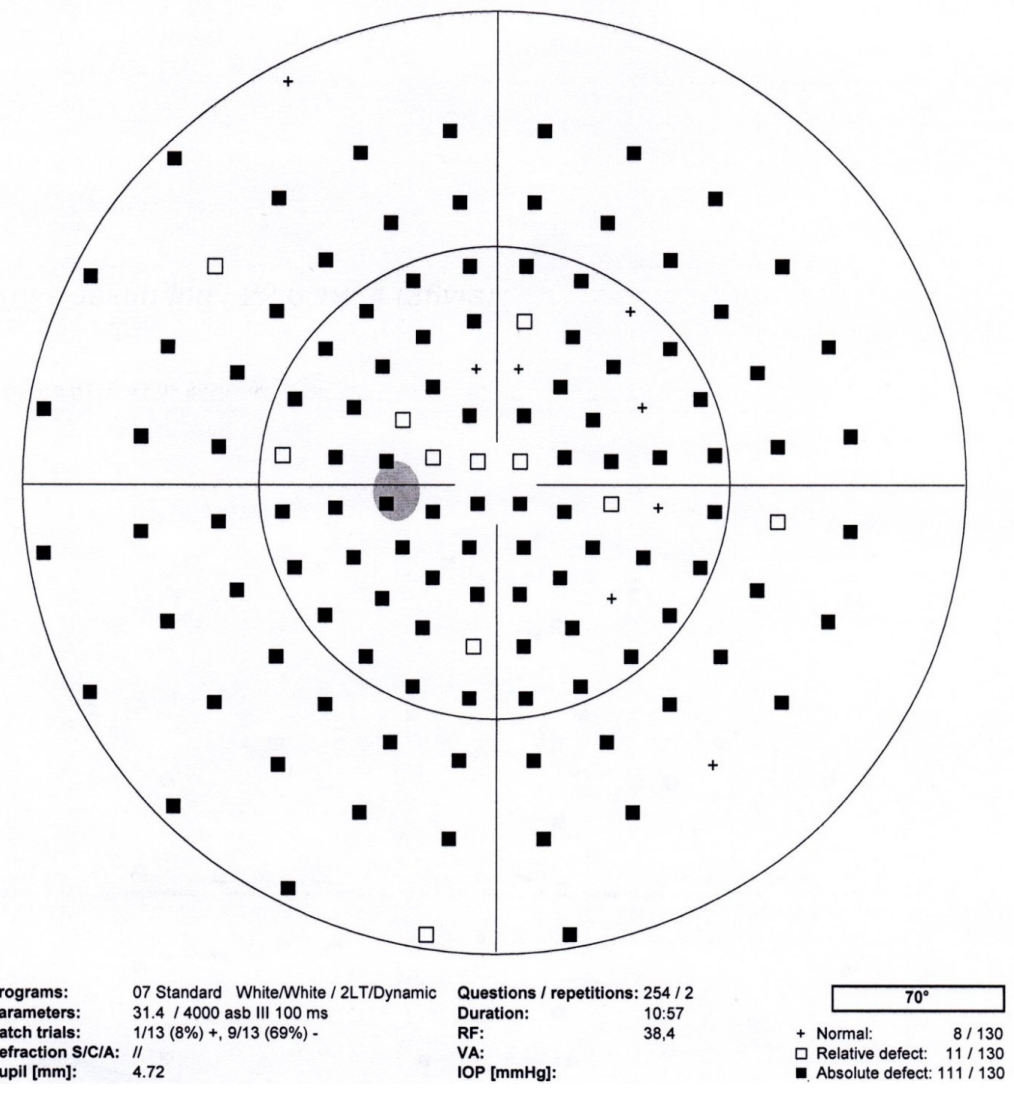

Fig. 6. Visual field examination of the left eye from 22 September 2020. A small remnant of the visual field in the upper half in the centre and between the centre and the blind spot with reduced sensitivity (denoted by the symbol $\square$ ), an isolated small upper paracentral remnant with preserved sensitivity (symbol +). 

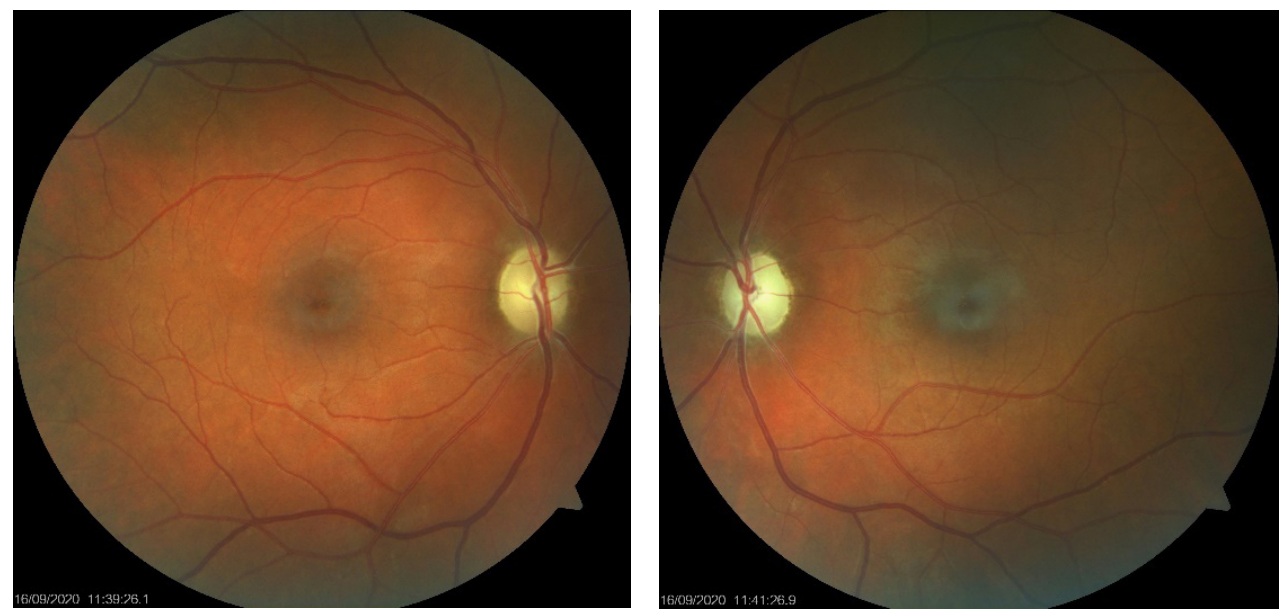

Fig. 7. Photograph of the fundus of the right and the left eye (right eye on the left side).

was without edema and with retinal pigment epithelium changes and fine drusen, the arteries were slightly narrowed (Fig. 7).

Angio OCT RTVue XR Avanti (Optovue, Inc., Fremont, CA, USA) showed a significant decrease in peripapillary vessel density on the right eye down to $35.7 \%$ (all) and $28.3 \%$ (small vessels). For the left eye the readings were $30.5 \%$ (all) and $23.4 \%$ (small vessels). We also recorded a similar significant decrease in RNFL, peripapillary $49 \mu \mathrm{m}$ for the right eye and $43 \mu \mathrm{m}$ for the left.

An electrophysiological examination conducted by means of a Roland Consult Retiscan instrument, according to ISCEV methodology, showed normal amplitudes on the right pattern electroretinogram (PERG). On the left, the amplitudes were pathologically reduced (P50-N95: $13.3 \mathrm{uV}$ and $9.1 \mathrm{uV}$, respectively). The N95 latency was prolonged to $112 \mathrm{~ms}$ on both sides. Visual evoked potential (VEP) when stimulated with larger squares showed a decrease in amplitude on the right, and a significant decrease in amplitude on the left (N75-P100: $7.8 \mathrm{uV}$ and 1.3 $\mathrm{uV}$, respectively). When using smaller square stimulation, the finding was similar ( $7.8 \mathrm{uV}$ and $2.9 \mathrm{uV}$, respectively). The P100 latency was not prolonged ${ }^{12}$.

Structural magnetic resonance imaging (MRI) examinations of the brain were performed on the following dates and devices: on 21 June 2019 with a 1.5T Magnetom Area, 10 channel coil Area Head / Neck 20 (Fig. 8); on 28 August 2019 with a 1.5T Magnetom Avanto, 12 channel coil Avanto Head Matrix (Fig. 9) (both devices from Siemens Healthcare GmbH, Erlangen, Germany); and on 16 September 2020 with a 3T Achieva dStream TX SERIES (Philips Healthcare, Best, The Netherlands) using a 32 channel SENSE RF head coil (Fig. 10).

Functional magnetic resonance imaging (fMRI) was conducted on a Philips Achieva TX SERIES system with a 3 Tesla magnetic field. A 6-channel SENSE RF head coil was used for scanning and later a 32-channel SENSE RF head coil was used. Optical stimulation during fMRI measurements was performed with an alternated inversion of a black and white checkerboard with a frequency of $2 \mathrm{~Hz}$. Measurements (during separate stimulation of the left and right eyes and subsequently both at the same time) contained 5 blocks of stimulation (duration 30 seconds), when the crosshairs were projected into the visual field, interspersed with equal blocks of rest. Each measurement consisted of 100 dynamic scans of EPI gradient echo sequences with the following basic parameters: $\mathrm{TR}=3 \mathrm{~s}, \mathrm{TE}$ $=30 \mathrm{~ms}$, spatial resolution $2 \times 2 \times 2 \mathrm{~mm} 3$.

The fMRI evaluation was performed with SPM 12 software using general linear model statistics and standard data preprocessing (motion correction, spatial normalization, and smoothing with a $4 \times 4 \times 4 \mathrm{~mm}$ core). The resulting statistical maps were thresholded at a significance level of $p=0.05$ with correction for multiple Family Wise Error (FWE) observations. The extent of activation was then assessed by the number of statistically significant voxels using the indicated statistical threshold, i.e. voxels where there is a high probability of activation of brain tissue for a given stimulation (Fig. 11).

\section{DISCUSSION}

Optic disc edema is caused by blockade of axoplasmic transport by nerve fibres. The cause may be a blood flow disorder at the level of the lamina cribriformis, intracranial hypertension, or compression at the level of the anterior part of the optic nerve.

The patient developed the first signs of edema of the optic discs and maculas three months after allogeneic transplantation. Perimetry showed bilateral concentric narrowing of the visual fields, on the right to 30 degrees and on the left to 5 degrees paracentrally. If it was edema of the optic discs, then the changes in the visual fields would be the enlargement of the blind spot. In the case of a compressive lesion, there would be an improvement in the visual fields after such a short time. Because the changes persisted, although they were smaller in comparison to the first examination, in terms of their etiology, we considered primarily the vascular component.

MRI of the brain showed no intracranial hypertension, but thickening of both optic nerves. Therefore, the ophthalmological diagnosis was determined as bilateral 

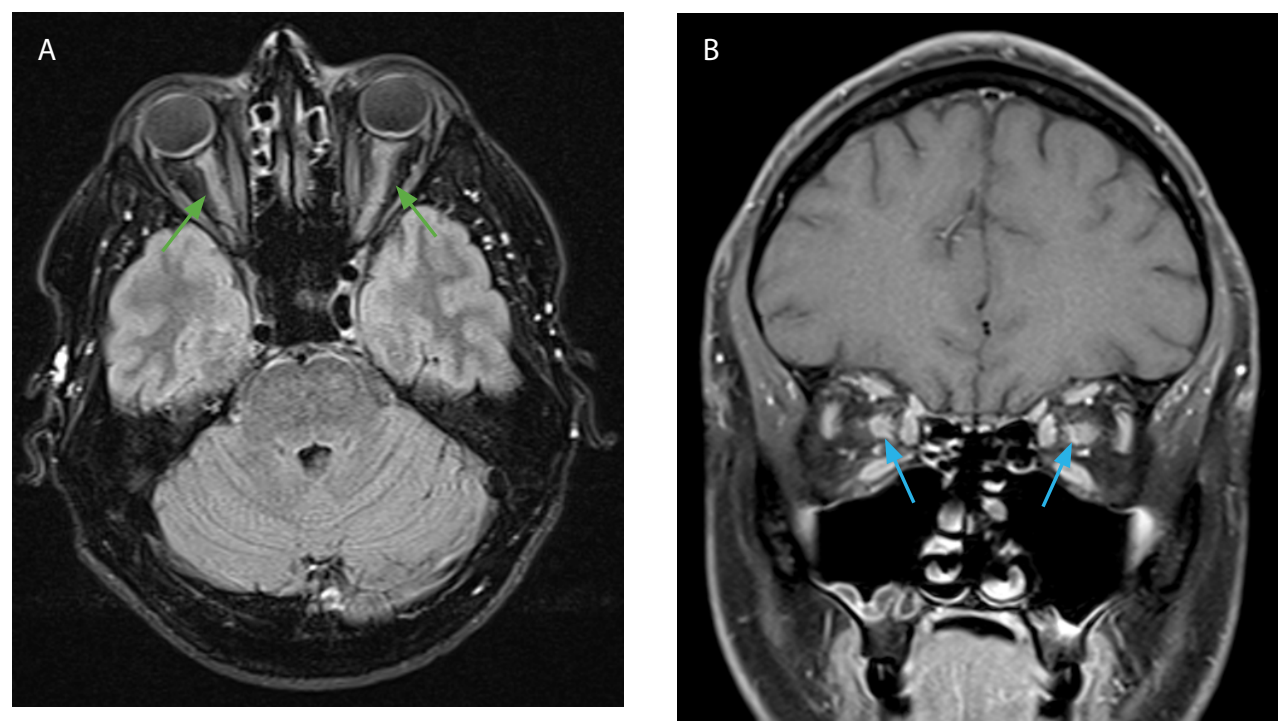

Fig. 8. MRI from 21 June 2019. Enlargement of the optic nerves on both sides. Nerve dilation intraorbitally throughout the course in FLAIR (A.) (green arrows). Nerve saturation after contrast agent application i.v., T1 mDIXON (B.) (blue arrows).
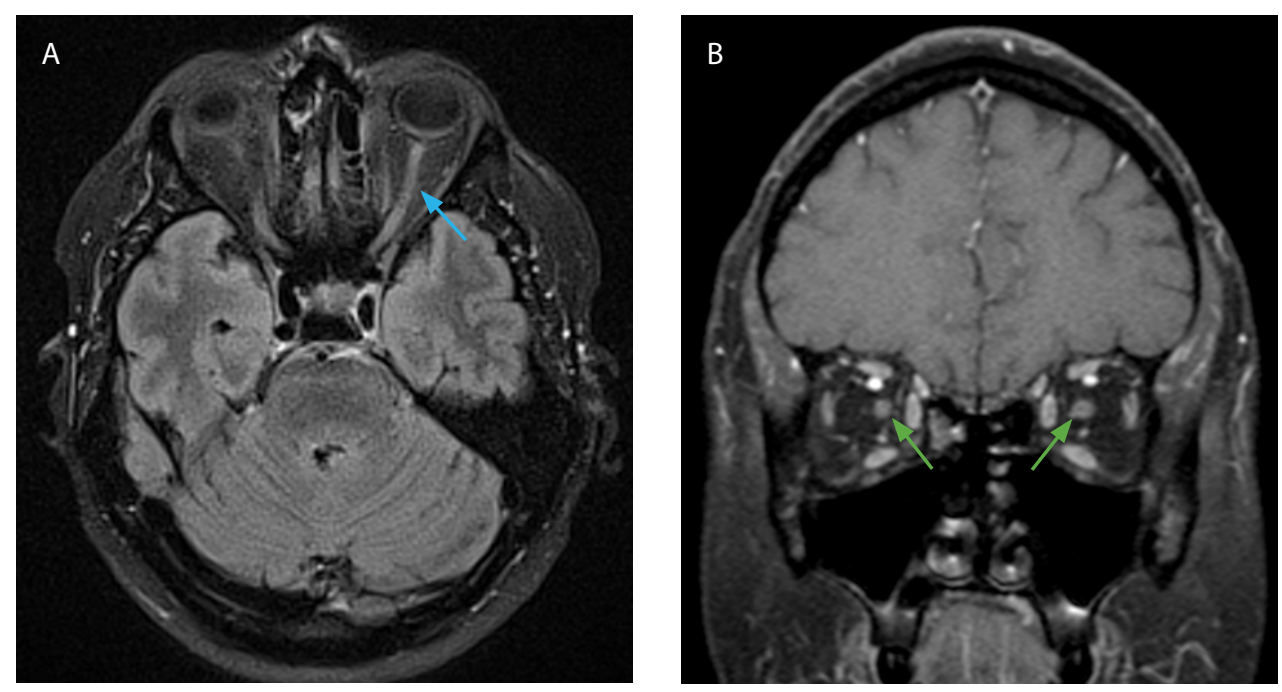

Fig. 9. MRI from 28 August 2019. Regression of optic nerve enlargement on both sides, FLAIR (A.) (blue arrow). No saturation after application of contrast agent i.v., T1 mDIXON (B) (green arrows).
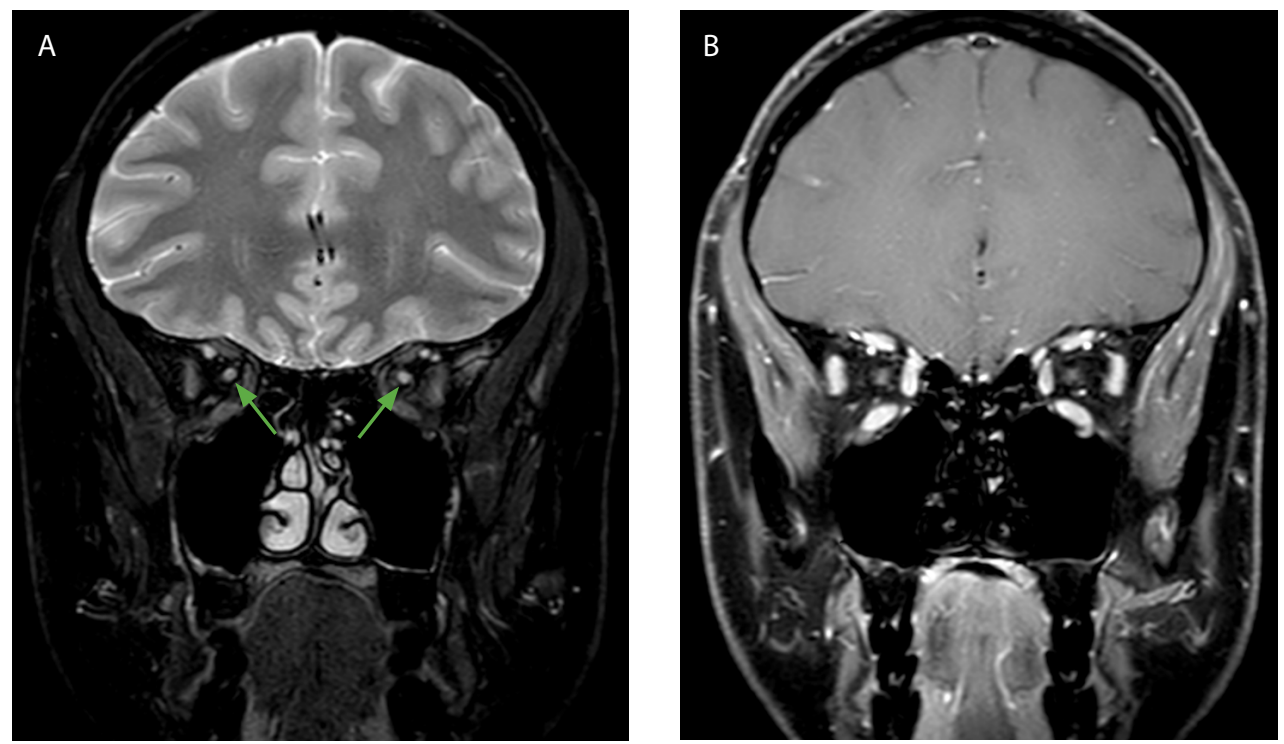

Fig. 10. MRI from 16 September 2020. Atrophy of optic nerves on both sides, T2 mDIXON (A.) (arrows). No saturation after application of contrast medium i.v., T1 mDIXON (B.). 

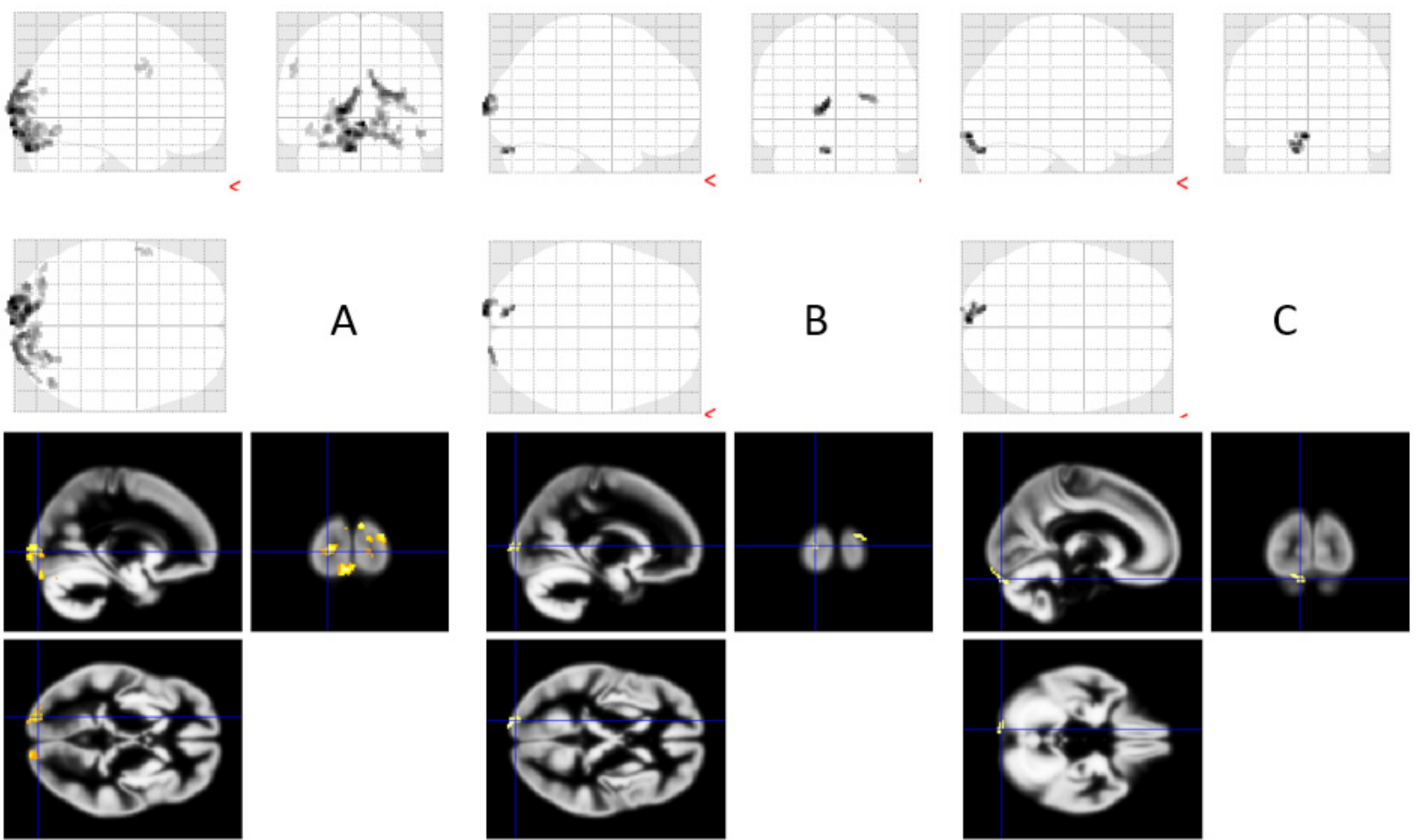
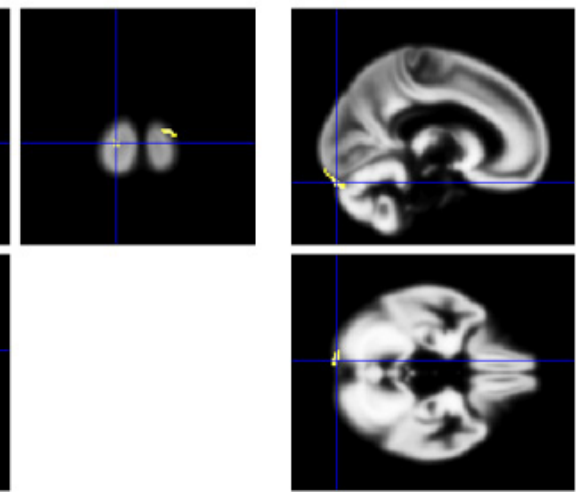

C

B

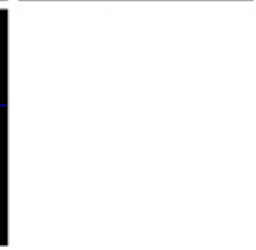

Fig. 11. Resulting activations in fMRI. The left column (A) shows the results for when both eyes were stimulated, the middle column (B) for the stimulation of the right eye and the left column (C) for the stimulation of the left eye. The total activations (using Maximum intensity projection) in sagittal, frontal and axial projection are shown in the upper part. The lower part shows cross-sections in the area of activation of the primary visual centre, again in sagittal, frontal and axial views. The number of statistically significant voxels in the stimulation of both eyes was 1250, while in the right eye it was only 117 and only 87 in the left. A statistical threshold of $P=0.001$ without correction for multiple observations (minimum number of voxels in cluster 10 ) was used in the evaluation.

intraocular and retrobulbar neuritis. The use of corticoids led to an improvement in the ocular findings.

We conclude that due to inflammatory changes, axoplasmic transport was blocked at the level of the optic discs. In addition, a circulatory disorder occurred, which in this case resulted in changes in the visual fields. The cause of the macular edema remains unclear. As with the optic nerve, we see its cause in a possible disorder of the vascular component.

An important clinical symptom of this patient was febrile periods that preceded visual impairment, both in the primary manifestation of the neuritis as well as in its recurrence at residual doses of corticoids. This fact could indicate that an infectious agent, especially an opportunistic viral infection, may have been involved in the etiopathogenesis of the clinical condition. It is important to note that due to the reactivation of HHV-6 before and soon after transplantation, the patient was pre-emptively treated with a virostatic agent (valganciclovir, cidofovir) the whole time from being discharged after transplantation. This could have reduced the likelihood of the detection of viral nucleic acids in the analysed biological materials.

HHV-6 infection in immunocompromised patients can cause optic neuritis. This phenomenon has been observed in patients with AIDS as well as in allogeneic transplant recipients. Despite maximal and often combined viro- static therapy (foscarnet, valganciclovir, cidofovir), the resulting therapeutic effect is largely dependent on the ability of the immune system to restore ${ }^{13,14}$.

HHV-6 reactivation in patients after allogeneic transplantation causes severe vascular endothelial damage and is an important etiopathogenetic component of HSCTrelated vascular complications such as TA-TMA ( ref. $^{15}$ ).

This fact could help to clarify the cause of the impairment of the vascular component involvement in our patient, despite no fully expressed TA-TMA after transplantation occurred. However, endothelial fibrinolysis would indicate endothelial dysfunction. Damage to the vascular component could also have been caused by possible formation of micro-thrombi, as there was repeated detection of lupus anticoagulans positivity. Congenital APC resistance aggravated the prothrombotic state, although retinal vein occlusion was not demonstrated.

Patients after allogeneic transplantation have a higher risk of thrombotic complications due to the vulnerability of the vascular endothelium ${ }^{16}$.

The patient did not develop the typical manifestations of GVHD, however, the detection of the autoantibodies ANA and ENA and of lupus anticoagulans could indicate the presence of autoimmune processes associated with a deregulated immune response and autoantibody formation often seen in the chronic form of GVHD (ref. ${ }^{17}$ ).

T-lymphocyte-mediated vascular damage in chronic 
GVHD can result in loss of vascular supply and impaired microcirculation in damaged tissues ${ }^{18}$.

The significant decrease in voxel activity after visual stimulation shown by fMRI, with a greater alteration after stimulation of the left eye indicates serious damage to the visual cortical structures as well. This corresponds to the PERG examination showing a more severe disorder in the left eye, as did the VEPs.

\section{CONCLUSION}

With this rare case study of a patient after allogeneic HSCT for B-ALL, we highlight possible differential diagnostic problems of optic disc edema and the central part of the retina of both eyes. We believe that these edemas were caused by a combination of several factors. First, inflammatory changes in the optic nerve sheaths led to a blockade of axoplasmic transport. Second, possible endothelial dysfunction of combined etiology along with microcirculation disorders, could have caused the impairment of the blood supply of the fibres of the optic nerve and also could have contributed to macular edema.

\section{ABBREVIATIONS}

ADV, adenovirus; ANA, antinuclear antibodies; APC, activated protein $\mathrm{C}$; B-ALL, B-cell acute lymphoblastic leukemia; BK virus, human polyomavirus, after the initials of that first patient in whom it was described; CMV, cytomegalovirus; CNS, central nervous system; CVA, central visual acuity; D, diopter; EBV, Epstein-Barr virus; ENA, extractable nuclear antigen antibodies; EPI, echo planar imaging; fMRI, functional magnetic resonance imaging; FWE, family wise error; GVHD, graft-versus-host disease; HHV-6, human herpes virus-6; HSTC, hematopoietic stem cell transplantation; HSV, herpes simplex virus; ISCEV, international society for clinical electrophysiology and vision; JC virus, human polyomavirus 2, John Cunningham virus; MRI, structural magnetic resonance imaging; OCT, optical coherence tomography; PB19, parvovirus B19; PCR, polymerase chain reaction; PERG, pattern electroretinogram; RNFL, retinal nerve fibre layer; TA-TMA, transplant-associated thrombotic microangiopathy; VD, vessel density; VEP, visual evoked potential; VZV, varicella-zoster virus.

Acknowledgement: Supported by MH CZ - DRO (FNOI, 00098892).

Author contributions: Authors contributed equally.

\section{REFERENCES}

1. Luft T, Benner A, Terzer T, Jodele S, Dandoy CE, Storb R, Kordelas L, Beelen D, Gooley T, Sandmaier BM, Sorror M, Zeisbrich M, Radujkovic A, Dreger P, Penack O. EASIX and mortality after allogeneic stem cell transplantation. Bone Marrow Transplant 2020;55(3):553-61.
2. Luft T, Dietrich S, Falk C, Conzelmann M, Hess M, Benner A, Neumann F, Isermann B, Hegenbart U, Ho AD, Dreger P. Steroid-refractory GVHD: T-cell attack within a vulnerable endothelial system. Blood 2011;118(6):1685-92.

3. Takatsuka H, Wakae T, Mori A, Okada M, Fujimori Y, Takemoto Y, Okamoto T, Kanamaru A, Kakishita E. Endothelial damage caused by cytomegalovirus and human herpesvirus-6. Bone Marrow Transplant 2003;31(6):475-9.

4. Dietrich S, Falk CS, Benner A, Karamustafa S, Hahn E, Andrulis M, Hegenbart U, Ho AD, Dreger P, Luft T. Endothelial vulnerability and endothelial damage are associated with risk of graft-versus-host disease and response to steroid treatment. Biol Blood Marrow Transplant 2013;19(1):22-7.

5. Balaguer-Rosello A, Bataller L, Piñana JL, Montoro J, Lorenzo I, Villaba A, Freiria C, Sevilla T, Muelas N, GuerreiroM, Carretero C, Gómez I, Solvez P, Ángel M, Sanz G, Sanz J. Noninfectious Neurologic Complications after Allogeneic Hematopoietic Stem Cell Transplantation. Biol Blood Marrow Transplant 2019; 25(9):1818-24.

6. Maffini E, Festuccia M, Brunello L, Boccadoro M, Giaccone L, Bruno B. Neurologic Complications after Allogeneic Hematopoietic Stem Cell Transplantation. Biol Blood Marrow Transplant 2017; 23(3):388-97.

7. Zheng X, Huang Y, Wang Z, Yan H, Pan S, Wang H. Presumed cytomegalovirus associated retrobulbar optic neuritis in a patient after allogeneic stem cell transplantation. Transpl Infect Dis 2012;14:17779.

8. Patel A, Olavarria E. Optic neuritis preceding progressive outer retinal necrosis in an immunocompromised patient after allogeneic stem cell transplantation. Ann Hematol 2013; 92(10):1427-29.

9. Yoshikawa T, Yoshida J, Hamaguchi M, Kubota T, Akimoto S, Ihira M, Nishiyama Y, Asano Y. Human herpesvirus 7 - associated meningitis and optic neuritis in a patient after allogeneic stem cell transplantation. J Med Virol 2003;70(3):440-43.

10. Moesen I, Kidd DP. Bilateral Inflammatory Optic Neuropathy Related to Graft versus Host Disease Following Allogeneic Bone Marrow Transplantation for Hodgkin Disease. Neuroophthalmology 2014;38(4):224-29.

11. Stefanou MI, Bischof F. Central and peripheral nervous system immune-mediated demyelinating disease after allogeneic hematopoietic stem cell transplantation. J Neuroimmunol 2017;307:74-81.

12. Lešták J, Nutterová E Pitrová Š, Krejčová $H$, Bartošová L, Forgáčová V. High tension versus normal tension glaucoma. A comparison of structural and functional examinations. J Clinic Exp Ophthalmol 2012,S5:006

13. Méchaï F, Boutolleau D, Manceron V, Gasnault J, Quertainmont Y, Brosseau JP, Delfraissy JF, Labetoulle M, Goujard C. Human herpesvirus 6-associated retrobulbar optic neuritis in an HIV-infected patient: response to anti-herpesvirus therapy and long-term outcome. J Med Virol 2007;79(7):931-34.

14. Hino Y, Doki N, Sekiya N, Takaki Y, Ohashi K. Optic neuritis as an initial manifestation of human herpesvirus 6 reactivation after unrelated bone marrow transplantation. Br J Haematol 2016;172(5):654-54.

15. Takatsuka H, Wakae T, Mori A, Okada M, Fojimori Y, Takemoto Y, Okamoto T, Kanamura A, Kakishita E. Endothelial damage caused by cytomegalovirus and human herpesvirus-6. Bone Marrow Transpl 2003;31:475-79.

16. Gavriilaki E, Sakellari I, Anyfanti P, Batsis I, Mallouri D, Psomopoulos F, Tsagiopoulou M, Koutra M, Yannaki E, Papalexandri A, Taylor P, Nikolousis E, Stamouli M, Holbro A, Baltadakis I, Liga M, Spyridonidis A, Tsirigotis P, Charchalakis N, Tsakiris DA, Brodsky RA, Passweg J, Stamatopoulos K, Anagnostopoulos A. Assessment of Endothelial Injury and Pro-Coagulant Activity Using Circulating Microvesicles in Survivors of Allogeneic Hematopoietic Cell Transplantation. Int J Mol Sci 2020;21:9768. doi: 10.3390/ijms21249768

17. Patriarca F, Skort C, Sporotto A, Zaja F, Falleti E, Mestroni R, Kikic F, Calistri E, Filì C, Geromin A, Cerno M, Fanin R. The development of autoantibodies after allogeneic stem cell transplantation is related with chronic graft-vs-host disease and immune recovery. Exp Hematol 2006;34(3):389-96.

18. Biedermann BC, Sahner S, Gregor M, Tsakiris DA, Jeanneret C, Pober JS, Gratwohl A. Endothelial injury mediated by cytotoxic T lymphocytes and loss of microvessels in chronic graft versus host disease. Lancet 2002;359(9323):2078-83. 